LAWRENCE LIVERMORE N A T IO N A L LABORATORY

Simulations Of Electron

Transport For Fast Ignition Using LSP

R. P. J. Town, C. Chen, L. A. Cottrill, M. H. Key, W. L. Kruer, A. B. Langdon, B. F. Lasinski, R. A. Snavely, C. H. Still, M. Tabak, S. C. Wilks, D. R. Welch

May 28, 2004

15th International Symposium on Heavy lon Inertial Fusion Princeton, NJ, United States June 7, 2004 through June 11, 2004 
This document was prepared as an account of work sponsored by an agency of the United States Government. Neither the United States Government nor the University of California nor any of their employees, makes any warranty, express or implied, or assumes any legal liability or responsibility for the accuracy, completeness, or usefulness of any information, apparatus, product, or process disclosed, or represents that its use would not infringe privately owned rights. Reference herein to any specific commercial product, process, or service by trade name, trademark, manufacturer, or otherwise, does not necessarily constitute or imply its endorsement, recommendation, or favoring by the United States Government or the University of California. The views and opinions of authors expressed herein do not necessarily state or reflect those of the United States Government or the University of California, and shall not be used for advertising or product endorsement purposes. 


\title{
SIMULATIONS OF ELECTRON TRANSPORT FOR FAST IGNITION USING LSP
}

\author{
R. P. J. Town ${ }^{*}$, C. Chen, L. A. Cottrill, M. H. Key, W. L. Kruer, A. B. Langdon, \\ B. F. Lasinski, R. A. Snavely, C. H. Still, M. Tabak, and S. C. Wilks \\ LLNL, 7000 East Ave, Livermore, CA, 94550-9234 \\ D. R. Welch
}

Mission Research Corp., 5001 Indian School Rd NE, Albuquerque, NM 87110-3946

\begin{abstract}
A crucial issue for the viability of the fast ignition approach to inertial fusion energy is the transport of the ignition pulse energy from the critical surface to the high-density compressed fuel. Experiments have characterized this transport through the interaction of short pulse, high intensity lasers with solid-density targets containing thin $\mathrm{K}_{\alpha}$ fluorescence layers. These experiments show a reasonably well-collimated beam, although with a significantly larger radius than the incident laser beam. We report on LSP calculations of these experiments, which show reasonable agreement with the experimental observations.
\end{abstract}

Keywords: Fast Ignition; Plasma simulation; Intense-particle beams; Laser light absorption in plasmas

PACS: 52.57.Kk; 52.65.-y; 52.59-f; 52.38Dx;

* Corresponding author. E-mail: town2@llnl.gov 


\section{Introduction}

In fast ignition ${ }^{1}$ a short-pulse high intensity laser is incident on a pre-compressed DT-fuel assembly. The laser light is deposited at the critical density (which is $1.1 \times 10^{21}$ $\mathrm{cm}^{-3}$ for $1-\mu \mathrm{m}$ laser light) generating relativistic electrons. These electrons propagate to the high-density $\left(10^{26} \mathrm{~cm}^{-3}\right)$ compressed fuel depositing their energy and igniting the fuel. Fast ignition offers a number of benefits compared to conventional "hot-spot” ignition (where the compression driver is carefully tailored to compress the fuel, but also has to form the ignition region). However, there are a number of technical and scientific uncertainties that must be addressed. The chief uncertainty (and major driver on the short-pulse igniter laser specification) is the coupling efficiency of the incident shortpulse laser to the fuel. There have been many active experimental campaigns to study electron generation and transport through surrogate solid planar targets ${ }^{2,3}$. In this paper we will present hybrid-PIC calculations using the LSP $\operatorname{code}^{4}$ of these transport experiments.

This paper is structured as follows: The experimental data is summarized briefly in section 2; The LSP code and the choice of the initial electron distribution function is described in section 3; Results from LSP calculations are presented in section 4; and conclusions are drawn in section 5.

\section{Review of the experimental data}

The two principal diagnostics that have been used to examine electron transport in short-pulse laser-matter interactions are both time integrated imaging diagnostics. The first technique looks at the XUV emission on the rear surface of the target. These XUV images are dominated by near-Planckian thermal emission, which for the measured $68 \mathrm{eV}$ 
photon energy should make the measurement sensitive to temperatures in the 10 to $100 \mathrm{eV}$ range. Shown in figure 1 is a typical XUV image of the rear surface of a $100 \mu \mathrm{m}$ thick Al foil irradiated by the 100-J, 1-ps Vulcan laser at the Rutherford Appleton Laboratory (RAL), UK recently obtained by Key and co-workers ${ }^{5}$. Work is ongoing to convert this into a temperature map. First the XUV images must be converted into absolute brightness. In order to relate the integrated absolute brightness to the temperature a series of Lasnex calculations of isochorically heated $\mathrm{Al}$ targets need to be performed. Preliminary analysis suggests that the central $70-\mu \mathrm{m}$ (fwhm) spot reaches a temperature of approximately $30 \mathrm{eV}$.

The second technique images $\mathrm{Cu} \mathrm{K}_{\alpha}$ emission from buried layers of $\mathrm{Cu}$ in $\mathrm{Al}$ (or $\mathrm{CH})$ targets. The emission size as a function of depth can be obtained by varying the position of the buried fluor layer in the target. $\mathrm{K}_{\alpha}$ emission is a two-step process: first an incident electron ejects a K-shell electron and then an outer shell electron fills the vacancy and emits a $\mathrm{K}_{\alpha}$ photon. Thus this diagnostics tells us the spatial extent of the hot electron beam. Figure 2 shows a compendium of data for $\mathrm{K}_{\alpha}$ emission width as a function of target depth taken from Stephens et al. ${ }^{6}$. These data show a minimum spot diameter of about $70 \mu \mathrm{m}$ for a bare $25-\mu \mathrm{m}$ thick $\mathrm{Cu}$ foil target. The diameter increases in a $20^{\circ}$ halfangle cone with increasing thickness of the $\mathrm{Al}$ transport layer in front of the $\mathrm{Cu}$ fluor.

\section{Defining the initial electron distribution function}

In order to model the experiments described above we have used the LSP code originally developed by Mission Research Corporation for use in the ion beam fusion community. LSP is a fully three dimensional hybrid-PIC code capable of running in Cartesian or cylindrical geometries. It employs a direct implicit particle push (based on 
the algorithm developed by Friedman, Langdon and Cohen ${ }^{7}$ ). This algorithm enables larger time steps than conventional explicit PIC codes, which must operate on space and time scales given by the Debye length and plasma frequency, allowing solid density, colder plasmas to be modeled. Such plasmas are more collisional and so LSP incorporates inter- and intra-species collisions based on Spitzer collision frequencies. Finally electrons can be represented as kinetic, or fluid particles. Exchange between the two different types of electrons is user settable and is not based on any physical mechanisms (such as electron runaway). In the fluid description the electrons carry a temperature, which is advanced by a separate energy equation that greatly reduces the effect of numerical cooling. The net effect of these algorithms is to enable LSP to model larger, more dense plasmas for longer simulation times than explicit PIC codes.

However, in order to model the spatial and temporal parameters of the experiment it is not presently possible, in the same simulation, to model the details of the laserplasma interaction, which generate the hot electron beam. Also, the current version of LSP does not have the correct boundary conditions for electromagnetic waves. Instead we have applied scaling laws derived from small-scale explicit PIC simulations and experimental data to establish the hot electron parameters from the incident laser intensity. The short-pulse Vulcan laser intensity pattern consists of a $10-\mu \mathrm{m}$ (fwhm) central spot (with a peak intensity of $4.0 \times 10^{19} \mathrm{~W} / \mathrm{cm}^{2}$ ) that contains approximately $30 \%$ of the energy and a much lower intensity $\left(<10^{17} \mathrm{~W} / \mathrm{cm}^{2}\right)$ broad "halo". Thus the Vulcan laser, like other petawatt lasers around the world, can be approximated by the overlap of a narrow high intensity Gaussian and a low intensity broader Gaussian. To convert this laser intensity pattern into an electron distribution we perform the following steps. First, 
the amount of energy that the hot electrons have to carry away from the laser spot is calculated from the conversion efficiency from laser energy to electron energy using the local intensity and a fit to a compilation of experimental data on conversion efficiencies to electron energy at different intensities. These experimental data show a constant conversion efficiency of $10 \%$ below $10^{15} \mathrm{~W} / \mathrm{cm}^{2}$; above that threshold intensity and up to $10^{21} \mathrm{~W} / \mathrm{cm}^{2}$ the conversion efficiency, $\eta$, depends on the laser intensity, $I$, according to:

$$
\eta=0.000175 \times I\left(W / \mathrm{cm}^{2}\right)^{0.2661} .
$$

Next the average energy of the hot electrons is calculated from the scaling law experimentally determined by $\mathrm{Beg}^{8}$ relating the hot electron temperature, $T_{\text {hot }}$, to the irradiance of the laser $\left(I \lambda^{2}\right)$ according to:

$$
T_{\text {hot }}(\mathrm{MeV})=0.1\left(I \lambda^{2} / 10^{17} \mathrm{~W} / \mathrm{cm}^{2} \mu \mathrm{m}^{2}\right)^{1 / 3} .
$$

The beam current density profile can now simply be determined from the ratio of the total electron energy carried at a particular radius and the energy carried by one electron at that radius. Finally a constant-in-space thermal spread is added to the electron energy distribution. The magnitude of the thermal spread is one of the variables that will be adjusted in the next section to compare with the experimental data. We have also performed simulations using the pondermotive scaling law, $T_{\text {hot }}(\mathrm{MeV})=\left(I \lambda^{2} / 10^{19} \mathrm{~W} / \mathrm{cm}^{2} \mu \mathrm{m}^{2}\right)^{1 / 2}$, which predicts a smaller number of electrons with higher energy in the central laser spot compared to the Beg scaling.

\section{Simulation results}

We have performed simulations of the $100 \mathrm{~J}, 1$ ps Vulcan laser experiments reported by Key $^{5}$ and Stephens ${ }^{6}$. Using the procedure outlined in the previous section the calculated total absorbed energy was about 27J. The forward directed electron energy distribution 
peaked at about $2 \mathrm{MeV}$ in the center of the laser spot and rapidly dropped to a few hundred $\mathrm{keV}$ in the wings of the spot (from about 10 to $100 \mu \mathrm{m}$ ). The addition of a constant thermal spread leads to the electrons in the central laser spot to have less angular divergence than the electrons in the wings of the focal spot. The electrons were injected through the left hand boundary into a solid density $100-\mu \mathrm{m} \mathrm{Al}^{3+}$ plasma at $5 \mathrm{eV}$. The perfect conducting boundaries were located at least $50 \mu \mathrm{m}$ from the plasma on all sides apart from the injection boundary (where the plasma touched the boundary to prevent the target from charging up). All the simulations were two-dimensional using cylindrical (RZ) geometry.

For comparison with the $\mathrm{K}_{\alpha}$ data a buried $20-\mu \mathrm{m} \mathrm{Cu}{ }^{2+}$ object was inserted into the $\mathrm{Al}$ plasma to model the fluor layer. This object recorded the birth-positions of the $\mathrm{K}_{\alpha}$ photons (using cross sections calculated from ITS ${ }^{9}$ ) which could be transported using ITS to the detector. In the LSP code objects are perfect conductors consequently there was a large resistivity change across the interface between the $\mathrm{Al}$ plasma and the $\mathrm{Cu}$ object. As the hot electrons transited across this interface we observed a rapid growth in the azimuthal magnetic field to $10 \mathrm{MG}$ in agreement with the analytic theory of Bell ${ }^{10}$. However, when a $\mathrm{Cu}$ plasma was used instead of the $\mathrm{Cu}$ object, the resistivity jump across the interface was much smaller and so no large magnetic field growth was observed at the interface. This suggested that an accurate resistivity model is important to correctly model transport across interfaces and in the plasma. LSP uses Spitzer conductivity, which is only valid for high temperatures $(>100 \mathrm{eV}$ for $\mathrm{Al})$. We approximated the resistivity for $\mathrm{Al}$ by a simple function in which the resistivity is held fixed at the $100 \mathrm{eV}$ Spitzer value below $100 \mathrm{eV}$ and obeys Spitzer above $100 \mathrm{eV}$. Figure 3 
shows contour plots of the injected electron beam density at $1.6 \mathrm{ps}$ for a calculation using a) Spitzer conductivity and b) the "constant" conductivity model. There is a distinct difference in the shape of the beam propagation between the two calculations. In the Spitzer case the hot electron beam has formed into small filaments whereas the beam shows a more diffuse structure in the "constant" conductivity model calculation. This difference is a direct consequence of the low temperature conductivity model. In the Spitzer model when the first injected electrons cross the plasma they heat the background plasma in channels, increasing the conductivity in the channels, which encourages further injected electrons to propagate along these high conductivity paths. In the constant conductivity case no such high conductivity channels are formed and so the injected electrons do not form into such filamented structures. All subsequent simulations reported here use the constant conductivity model.

The $\mathrm{K}_{\alpha}$ imaging diagnostic gives time-integrated images of the emission generated by the hot electron beam. The diagnostic will record both $K_{\alpha}$ photons generated by the forward going hot electron beam and by backward going electrons that have been reflected, or "refluxed" at the rear surface. In fact, the trajectories of the hot electrons can be quite complex and show multiple reflections at both the front and back surfaces of the target. Figure 4 shows an example of the hot electron trajectories for particles initially injected at a radius of $10 \mu \mathrm{m}$ at around the peak of the laser pulse overlaid on a contour plot of the electric field in the z-direction. At early times the hot electrons traverse the target and exit the rear surface of the target. This creates a very large $\left(10^{9} \mathrm{~V} / \mathrm{cm}\right)$ rear surface electric field that is responsible for accelerating protons to high energies ${ }^{11}$ and causes subsequent hot electrons to be reflected or, in conjunction with the large rear-surface magnetic field, 
to run along the rear surface of the target. We analyzed the $\mathrm{K}_{\alpha}$ photon generation as a function of time in our LSP calculations. The overall structure of the birth-position of the photons as a function of radius remained relatively constant as a function of time suggesting that the time integrated diagnostic is a good measure of the forward going transport of the hot electron beam. This has been experimentally confirmed by using targets with thicker rear layers of $\mathrm{Al}$ (points marked with an "X" in figure 2) that show similar emission radii as the standard $100 \mu \mathrm{m} \mathrm{Al}$ targets.

In order to determine the sensitivity of the emission region to the scaling laws mentioned in section 3 we performed a number of calculations using different amounts of thermal spread. We found very little difference in the $K_{\alpha}$ emission spot size between the Beg and pondermotive scaling laws when the $\mathrm{Cu}$ object was buried $30 \mu \mathrm{m}$ into the Al plasma. There was greater sensitivity to the initial thermal spread; for example, the emission diameter increased by about $25 \%$ when the thermal spread was increased from 200 to $300 \mathrm{keV}$ and increased a further $20 \%$ when the thermal spread was increased to $600 \mathrm{keV}$. We found reasonable agreement with the experimental data using an injected hot electron spectrum based on the Beg scaling with a thermal spread of $300 \mathrm{keV}$.

Finally we compared the rear surface temperature profile with the XUV imaging diagnostic. The XUV diagnostic is sensitive to the peak temperature so the LSP calculation was advanced for 6 ps until the temporal temperature peak in all of the rearsurface cells had been reached. This peak was recorded for each cell and corrected for real equation of state effects (LSP uses an ideal gas equation of state which over predicts the temperature by about a factor of 3). The LSP calculation predicts a peak temperature of $35 \mathrm{eV}$ in an approximate $70-\mu \mathrm{m}$ spot surrounded by a cooler $10 \mathrm{eV}$ pediment. The LSP 
calculation shows good agreement with the width of the central XUV image when the injected electrons used Beg scaling and a thermal spread of $300 \mathrm{keV}$. Work continues to establish the experimental temperature.

\section{Conclusions}

In this paper we have presented results of LSP calculations of recent electron transport experiments. We have shown that the electron transport was sensitive to the conductivity model used. In particular the filamentation of the electron beam was reduced when a more realistic “constant” conductivity was employed. We also showed reasonable agreement between LSP calculations and both the $\mathrm{K}_{\alpha}$ and XUV imaging diagnostics. However, the initial injected electron energy and density distribution was based on simple scaling laws rather than first principles calculations. To address this issue we plan to couple radiation-hydrodynamics, explicit PIC, and LSP calculations together. The hydro-code will be used to predict the amount of pre-formed plasma that is created when the laser pre-pulse interacts with the solid target. The explicit PIC calculations (which resolves the plasma frequency and small spatial scales) will then model the interaction of the laser intensity pattern with the pre-formed plasma to obtain the hot electron distribution. This distribution will then be transported by LSP in a manner similar to reported here. By coupling these three computational techniques together we will be able resolve and simulate in a reasonable time the essential physics necessary to determine the coupling of the short-pulse laser to the compressed fuel. 


\section{Acknowledgments}

This work was performed under the auspices of the U.S. Department of Energy by

University of California, Lawrence Livermore National Laboratory under Contract W7405-Eng-48. 


\section{Figures}

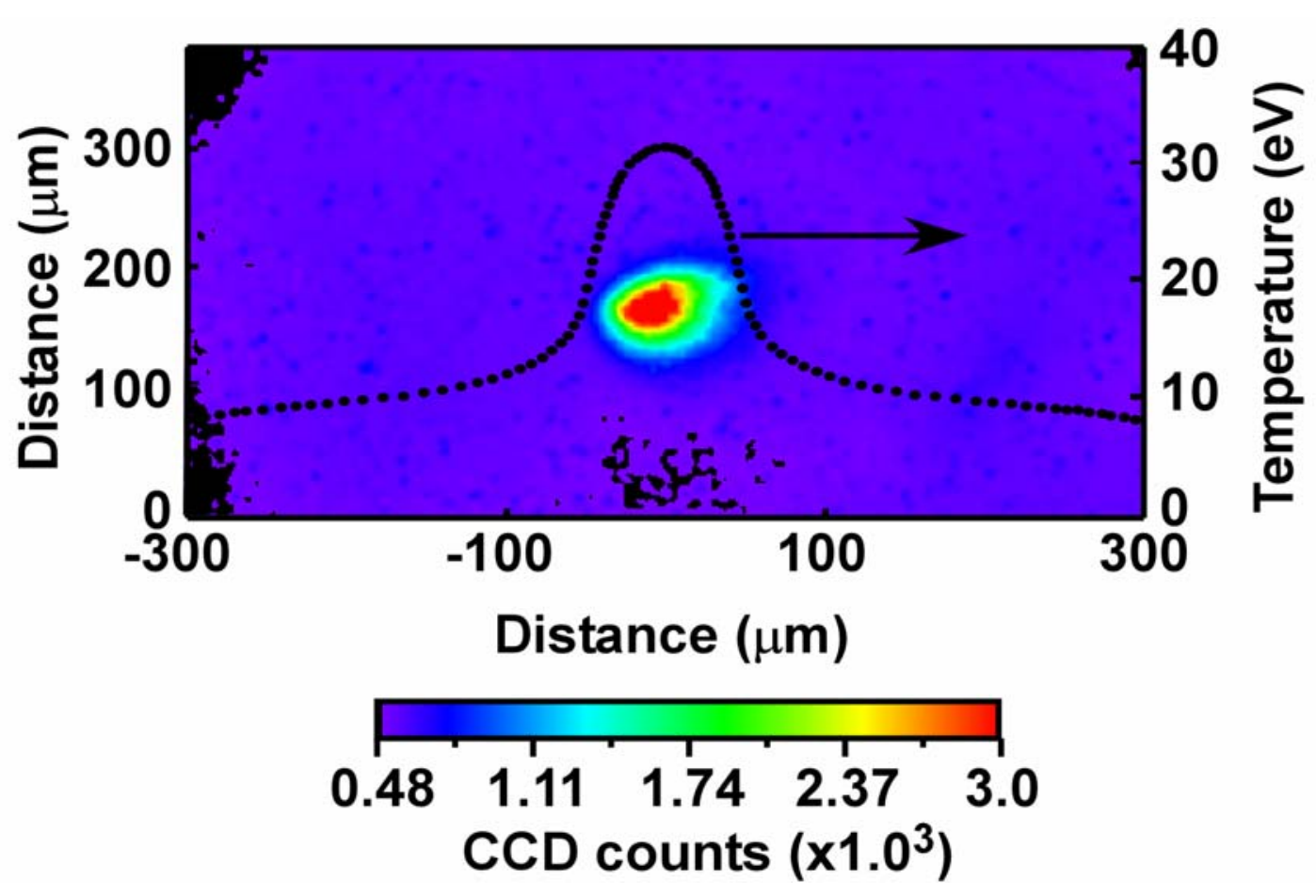

Fig. 1. A contour plot of an XUV image of the rear surface of a $100 \mu \mathrm{m} \mathrm{Al}$ foil illuminated by the short-pulse Vulcan laser, overlaid by the rear-surface temperature profile predicted by the LSP code (dots). 


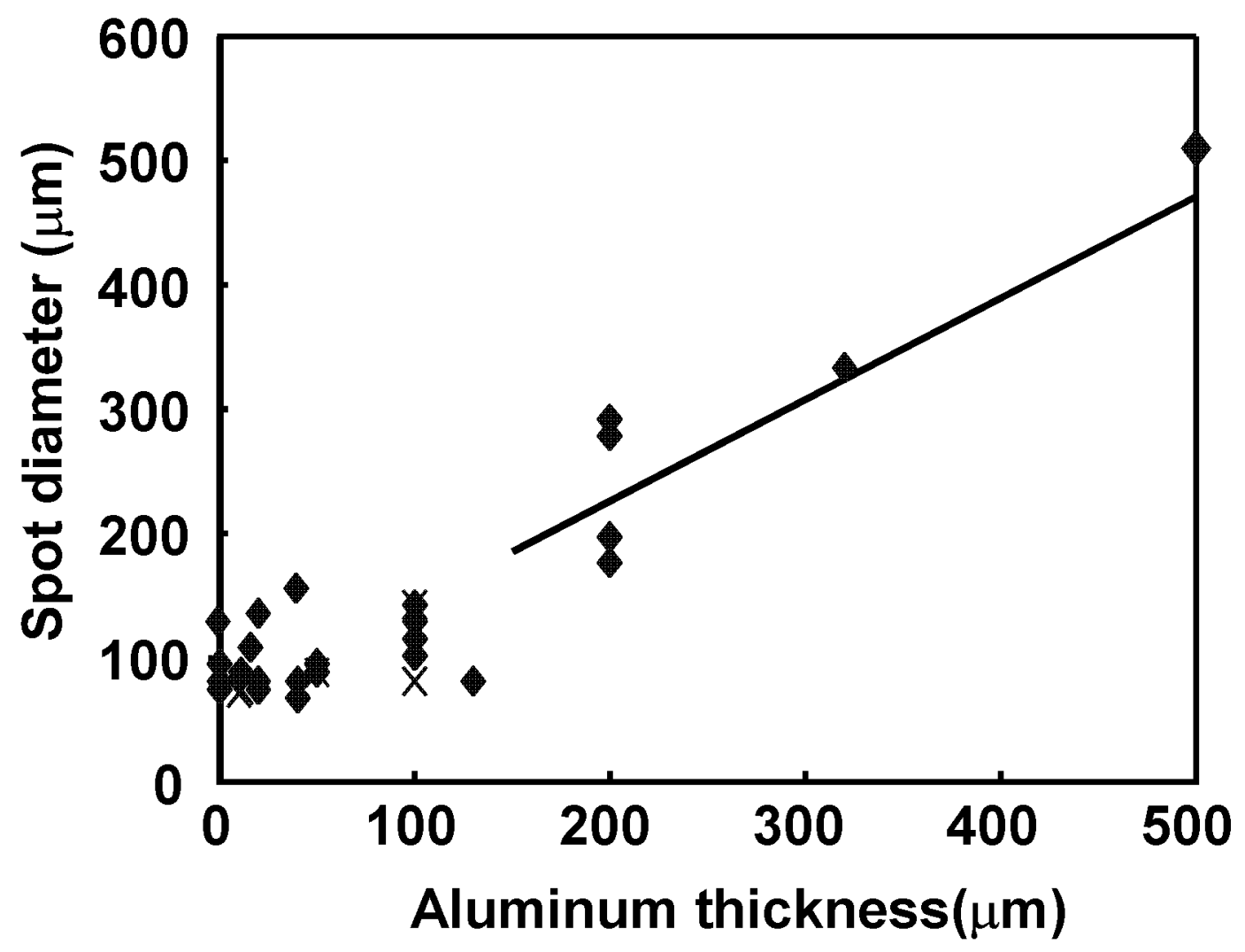

Fig. 2. $K_{\alpha}$ spot diameter at half-maximum intensity as a function of Al thickness. The line is a linear fit to that data, showing a spreading half-angle of $\sim 20^{\circ}$. The $X$ 's indicate targets with thicker back layers to limit refluxing. (Modified from Stephens et al. ${ }^{6}$ ) 
UCRL-PROC-204414

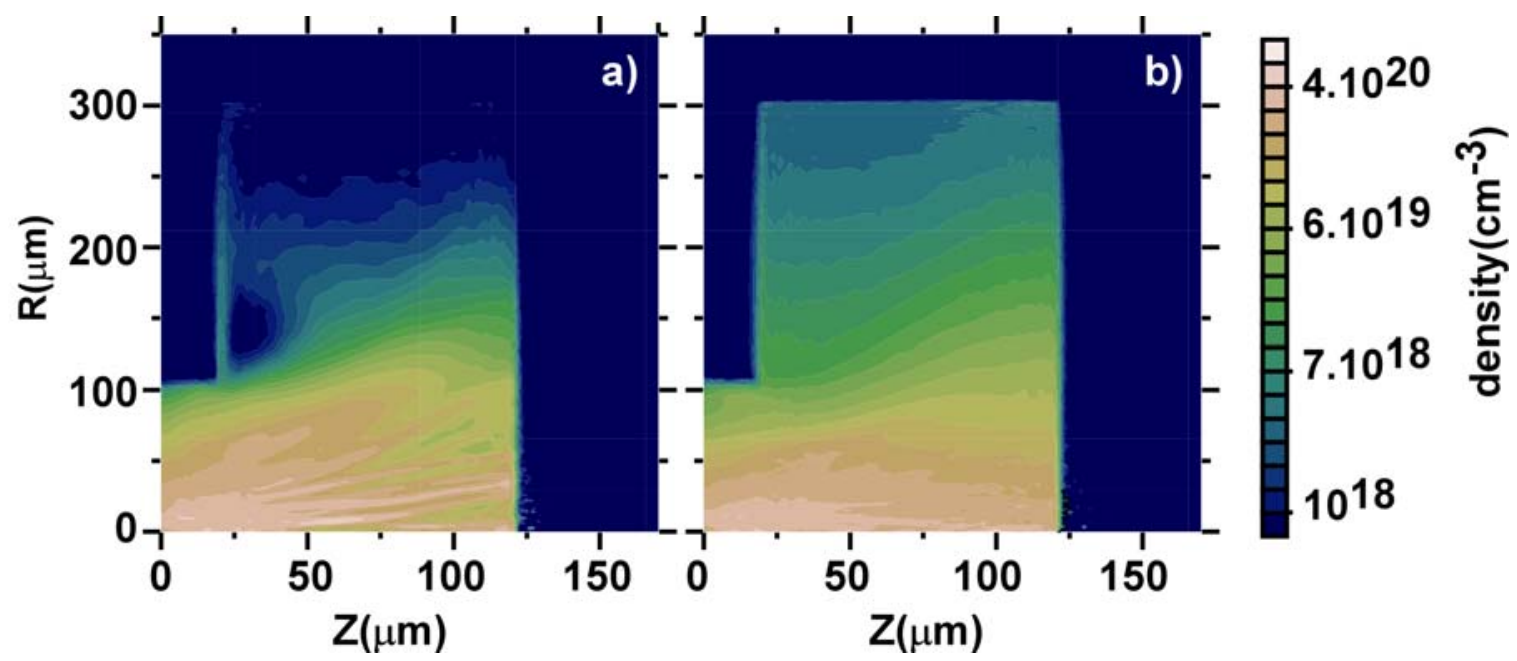

Fig. 3. The injected beam density at $1.6 \mathrm{ps}$ for a calculations using a) Spitzer conductivity and b) the "constant" conductivity model. 


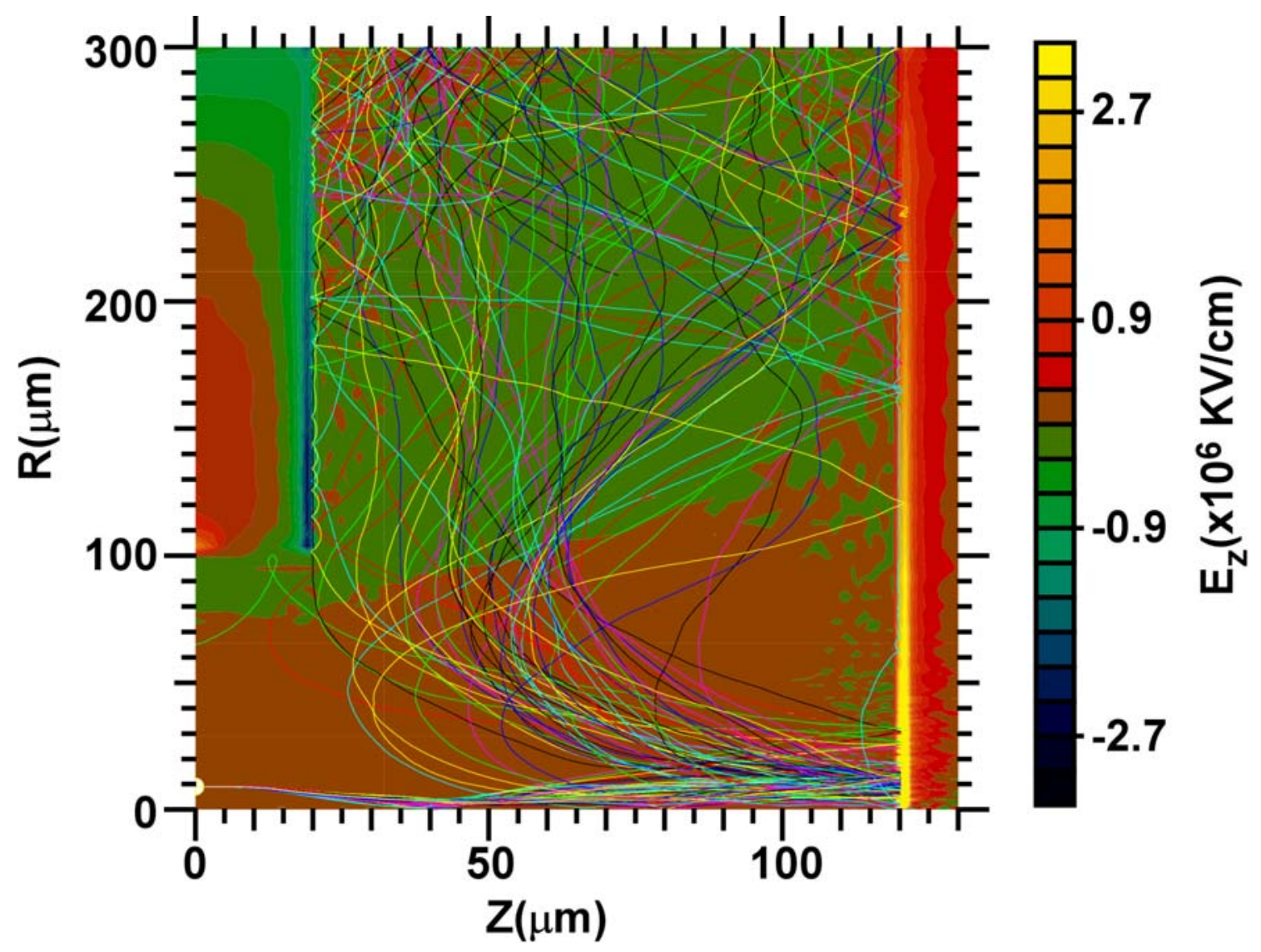

Fig.4. Trajectories of electrons initially injected at a radius of $10 \mu \mathrm{m}$ on top of color contours of the electric field component in the z-direction. 


\section{References}

${ }^{1}$ M. Tabak, J. Hammer, M. E. Glinsky, W. L. Kruer, S. C. Wilks, J. Woodworth, E. M. Campbell, M. D. Perry, and R. J. Mason, Phys. Plasmas 1, 1626 (1994).

${ }^{2}$ J. A. Koch, M. H. Key, R. R. Freeman, S. P. Hatchett, R. W. Lee, D. Pennington, R. B. Stephens, M. Tabak, Phys. Rev. E: 65, 016410 (2002).

${ }^{3}$ F. Amiranoff, S. D. Baton, L. Gremillet, O. Guilbaud, M. Koenig, E. Martinolli, J. J. Santos, M. R. Le Gloahec, C. Rousseaux, T. Hall, D. Batani, A. Bernardinello, G. Greison, E. Perelli, F. Scianitti, M. H. Key, J. A. Koch, A. J. Mackinnon, R. R. Freeman, R. A. Snavely, C. Andersen, T. E. Cowan, R. B. Stephens, Y. Aglistkiy, American Institute of Physics Conference Proceedings, 634, 28 (2002).

${ }^{4}$ D. R. Welch, D. V. Rose, B. V. Oliver, and R. E. Clark, Nucl. Inst. Meth. Phys. Res. A 464, 134 (2001).

${ }^{5}$ M. H. Key, F. Amiranoff, C. Andersen, S. D. Batani, S.D. Baton, T. Cowan, N. Fisch, R. R. Freeman, L.Gremillet, T. Hall, S. P. Hatchett, J. M. Hill, J. A. King, R Kodama, J. A. Koch, M. Koenig, B. Lasinski, B. Langdon, A. J. MacKinnon, E. Martinolli, P. Norreys, P. Parks, E. Perelli-Cippo, M. Rabec Le Gloahec, M. Rosenbluth, C. Rousseaux, J. J. Santos, F. Scianitti, R. Snavely, M. Tabak, K Tanaka, R. P. J. Town , T. 
Tsutumi, and R. B. Stephens, in Inertial Fusion Sciences and Applications 2003, edited by K. Tanaka, D. D. Meyerhofer, and J. Meyer-ter-Vehn (Elsevier, Paris, 2004).

${ }^{6}$ R. B. Stephens, Y. Aglitskiy, F. Amiranoff, C. Andersen, D. Batani, S. D. Baton, T. Cowan, R. R. Freeman, T. Hall, S. P. Hatchett, J.M. Hill, M.H. Key, J. A. King, J. A. Koch, M. Koenig, A. J. MacKinnon, K. L. Lancaster, E. Martinolli, P. Norreys, E. Perelli-Cippo, M. Rabec Le Gloahec, C. Rousseaux, J. J. Santos, F. Scianitti, R.A. Snavely, accepted for publication in Phys. Rev. E: (2004).

${ }^{7}$ A. Friedman, A. B. Langdon, and B. I. Cohen, Comments on Plasma Physics \& Controlled Fusion 6, 225 (1981); D. W. Hewett and A. B. Langdon, J. Comp. Phys. 72, 121 (1987).

${ }^{8}$ F. N. Beg, A. R. Bell, A. E. Dangor, C. N. Danson, A. P. Fews, M. E. Glinsky, B. A. Hammel, P. Lee, P. A. Norreys, and M. Tatarakis, Phys. Plasmas 4, 447 (1997).

${ }^{9}$ J. A. Halbleib, R. P. Kensek, G. D. Valdez, S. M. Seltzer, and M. J. Berger, IEEE Trans. Nucl. Sci. NS-39, 1025 (1992).

${ }^{10}$ A. R. Bell, J. R. Davies and S. M. Guerin, Phys. Rev. E: 58, 2471 (1998).

${ }^{11}$ S. P. Hatchett, C. G. Brown, T. E. Cowan, E. A. Henry, J. S. Johnson, M. H. Key, J. A.

Koch, A. B. Langdon, B. F. Lasinski, R. W. Lee, A. J. Mackinnon, D. M. Pennington, M. D. Perry, T. W. Phillips, M. Roth, T. C. Sangster, M. S. Singh, R. A. Snavely, M. A. Stoyer, S. C. Wilks, and K. Yasuike, Phys. Plasmas 7, 2076 (2000) 
UCRL-PROC-204414 